\title{
Experience of Manual Compression Hemostasis Under Endoscopic Observation for Acute Hemorrhagic Rectal Ulcer
}

\author{
Hideaki Kawabata $^{\mathrm{a}, \mathrm{b}}$, Katsutoshi Yamaguchi ${ }^{\mathrm{a}}$, Yukino Kawakatsu ${ }^{\mathrm{a}}$, Yuki Ueda ${ }^{\mathrm{a}}$, \\ Yuji Okazaki ${ }^{\mathrm{a}}$, Misuzu Hitomi ${ }^{\mathrm{a}}$, Masatoshi Miyata ${ }^{\mathrm{a}}$, Shigehiro Motoi ${ }^{\mathrm{a}}$
}

\begin{abstract}
We experienced two cases in which manual compression hemostasis under endoscopic observation was used in patients with acute hemorrhagic rectal ulcer (AHRU). The patients experienced an episode of massive fresh hematochezia, requiring the blood transfusion. Emergent sigmoidoscopy revealed multiple ulcers with a large protuberant visible vessel or with gush-out hemorrhage on the lower rectum. Endoscopic hemostasis by hemoclips and hypertonic saline-epinephrine injection was attempted; however, mechanical mucosal injury induced by hemoclips and needles caused another gush-out hemorrhage. Thus, the site of bleeding was manually compressed by a forefinger under endoscopic observation. After $5 \mathrm{~min}$, compression hemostasis was achieved, and the postoperative course was uneventful. Manual compression hemostasis under endoscopic observation is useful and worth attempting for AHRU.
\end{abstract}

Keywords: Manual compression hemostasis; Acute hemorrhagic rectal ulcer; Endoscopy

\section{Introduction}

Acute hemorrhagic rectal ulcer (AHRU) is characterized by the sudden onset of painless massive bleeding from rectal ulcers, which commonly occurs in patients with serious underlying disorders [1-3]. The recent development of various endoscopic hemostatic techniques has contributed to reducing the indication of surgical treatments such as transanal suture ligation by up to $2-5 \%[1,3-5]$. However, we sometimes experience cases in which another hemorrhage is induced due to mechanical mucosal injury from a hemoclip or injection needle, or dete-

Manuscript submitted February 15, 2019, accepted March 14, 2019

aDepartment of Gastroenterology, Kyoto Okamoto Memorial Hospital, Kyoto, Japan

${ }^{\mathrm{b} C}$ Corresponding Author: Hideaki Kawabata, Department of Gastroenterology, Kyoto Okamoto Memorial Hospital, 58 Nishinokuchi, Sayama, Kumiyamacho, Kuze-gun, Kyoto 613-0034, Japan. Email: hkawabata@okamoto-hp.or.jp

doi: https://doi.org/10.14740/gr1162 rioration of the ulcer due to thermocoagulation.

We herein report two cases in which manual compression hemostasis under endoscopic observation was used in order to avoid excessive mucosal injury in patients with AHRU.

\section{Case Reports}

\section{Case 1}

The patient was a 77-year-old man with prostatic cancer who after receiving intensity-modulated radiotherapy suffered from right inguinal pain and leg edema due to a metastatic iliac lymph node which had increased in size. He was using etodolac and prednisolone. Three days after starting palliative radiotherapy for the metastatic lymph node, he experienced an episode of massive fresh hematochezia, and his hemoglobin level dropped from 9.8 to $6.3 \mathrm{~g} / \mathrm{dL}$, requiring the transfusion of 4 units of packed red blood cells. Emergent sigmoidoscopy revealed multiple ulcers with a large protuberant visible vessel on the lower rectum (Fig. 1a, b). The placement of a total of seven hemoclips was attempted; however, mechanical mucosal injury induced by the hemoclips caused gush-out hemorrhages, which occurred repeatedly (Fig. 1c). Thus, we inserted a forefinger and manually compressed the site of bleeding under endoscopic observation (Fig. 1d). After 5 min, compression hemostasis was achieved (Fig. 1e). The postoperative course was uneventful without massive bleeding or the need for blood transfusion until he died 3 weeks after the achievement of hemostasis due to the worsening of the primary disease.

\section{Case 2}

The patient was a 74-year-old woman who was bedridden due to cerebral infarction and subarachnoid hemorrhage. She was using clopidogrel (an antiplatelet agent). She experienced an episode of massive fresh hematochezia, and her hemoglobin level dropped from 14.7 to $8.6 \mathrm{~g} / \mathrm{dL}$, requiring the transfusion of 4 units of packed red blood cells. Emergent sigmoidoscopy revealed multiple ulcers with gush-out hemorrhage on the lower rectum (Fig. 2a). Two hemoclips and hypertonic saline-epinephrine (HSE) injection were attempted; however, mechani- 


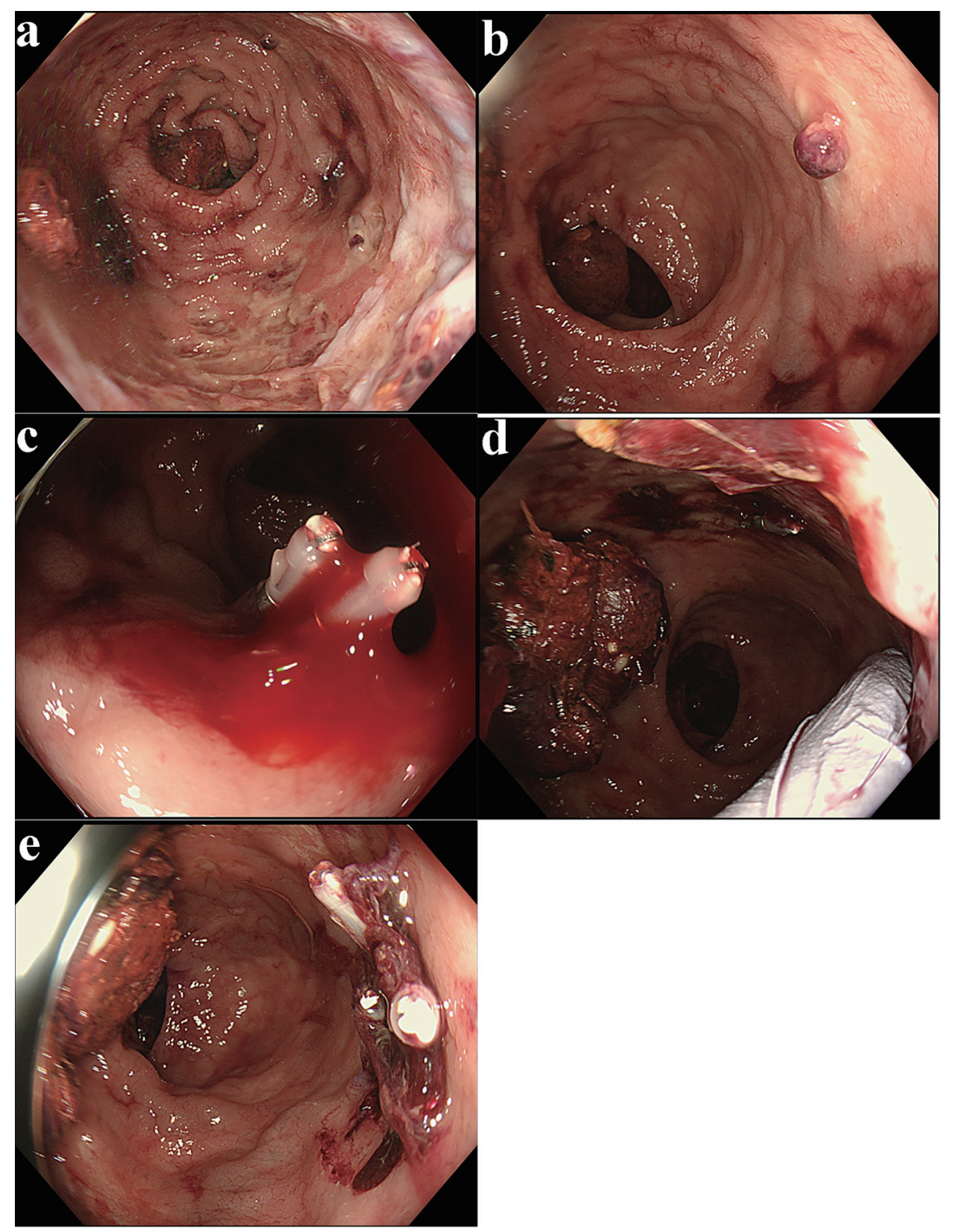

Figure 1. (a) Sigmoidoscopy revealed multiple ulcers on the lower rectum (case 1). (b) One of ulcers accompanied a large protuberant visible vessel (case 1). (c) Mechanical mucosal injury induced by the hemoclips caused gush-out hemorrhages, which occurred repeatedly (case 1). (d) The site of bleeding was manually compressed by a forefinger under endoscopic observation (case 1). (e) Compression hemostasis was achieved after 5 min (case 1).

cal mucosal injury induced by hemoclips and needles caused another gush-out hemorrhage (Fig. 2b). Thus, we manually compressed the site of bleeding under endoscopic observation (Fig. 2c). After 5 min, compression hemostasis was achieved (Fig. 2d). The postoperative course has been uneventful for 1 month after the achievement of hemostasis.

\section{Discussion}

We were able to achieve long-term hemostasis without surgical procedures using manual compression under endoscopic observation. In AHRU, the rectal mucosa and ulcers are assumed to be fragile and refractory because most patients are suffering from malnutrition due to an inadequate oral intake, atherosclerosis, the use of anti-coagulants, and the reduction of rectal blood flow due to being in the supine position $[1,4,5]$. Even in such condition, manual compression can achieve hemostasis, with no harmful effect (including mechanical, chemical and burn injury in endoscopic procedures) on the mucosa, and the long-term outcome is also favorable. Moreover, this technique is easy to perform and precise because we can compress the bleeding lesion with pinpoint accuracy under endoscopic observation. To our knowledge, this is the first report 


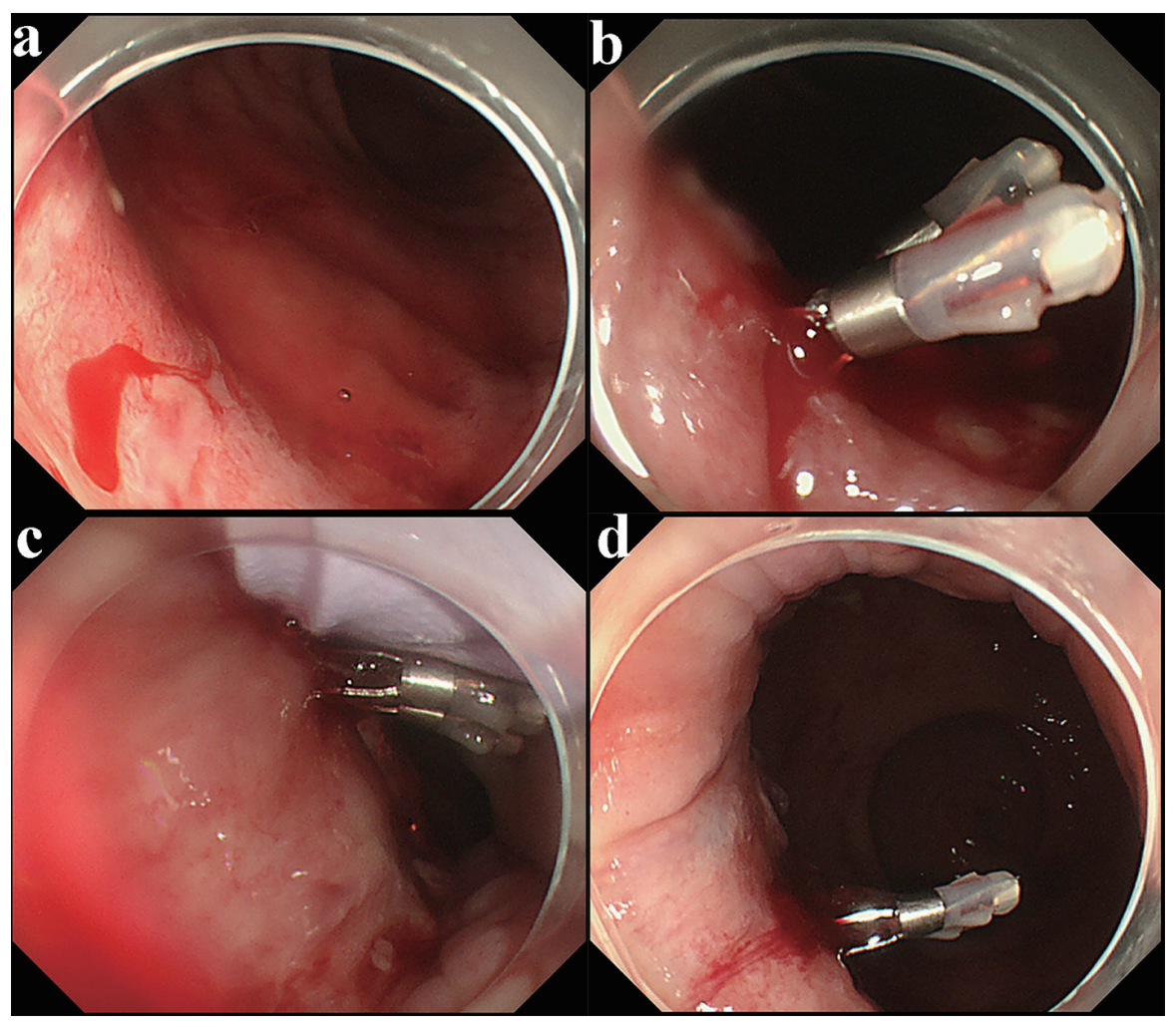

Figure 2. (a) Sigmoidoscopy revealed multiple ulcers with gush-out hemorrhage on the lower rectum (case 2). (b) Mechanical mucosal injury induced by hemoclips and needles caused another gush-out hemorrhage (case 2). (c) The site of bleeding was manually compressed by a forefinger under endoscopic observation (case 2). (d) Compression hemostasis was achieved after $5 \min ($ case 2).

showing its utility. It should be indicated in cases of oozing or gush-out hemorrhage, regardless of whether it is combined with an endoscopic procedure. This technique is also available as a primary temporal hemostatic procedure when bleeding is too active to perform endoscopic procedures. After using hemoclips, we should compress the bleeding point gently from the side of the hemoclips so as not to remove them.

In conclusion, manual compression hemostasis under endoscopic observation is useful and worth attempting for AHRU. However, more experience is needed to confirm its utility.

\section{Acknowledgments}

None.

\section{Conflict of Interest}

The authors declare that they have no conflict of interest.

\section{Financial Disclosure or Funding}

None.

\section{Informed Consent}

Written informed consent was obtained from the patient.

\section{Author Contributions}

H. Kawabata, K. Yamaguchi, Y. Kawakatsu, Y. Ueda, Y. Okazaki, M. Hitomi, and M. Miyata contributed to endoscopic diagnosis and treatment. M. Miyata and S. Motoi supervised the findings of this work. H. Kawabata wrote the manuscript with support from M. Hitomi and S. Motoi. All authors discussed the results and contributed to the final manuscript.

\section{References}

1. Tseng CA, Chen LT, Tsai KB, Su YC, Wu DC, Jan CM, Wang WM, et al. Acute hemorrhagic rectal ulcer syndrome: a new clinical entity? Report of 19 cases and review of the literature. Dis Colon Rectum. 2004;47(6):895903; discussion 903-895.

2. Hung HY, Changchien CR, You JF, Chen JS, Chiang JM, Yeh CY, Fan CW, et al. Massive hematochezia from acute hemorrhagic rectal ulcer in patients with severe comorbid illness: rapid control of bleeding by per anal sutur- 
ing of bleeder using anoretractor. Dis Colon Rectum. 2006;49(2):238-243.

3. Oku T, Maeda M, Ihara H, Umeda I, Kitaoka K, Waga E, Wada Y, et al. Clinical and endoscopic features of acute hemorrhagic rectal ulcer. J Gastroenterol. 2006;41(10):962970.

4. Matsumoto T, Inokuma T. Clinical course and rebleeding predictors of acute haemorrhagic rectal ulcer: 5-year experience and review of the literature. Colorectal Dis. 2013;15(7):878-884.

5. Motomura Y, Akahoshi K, Matsui N, Kubokawa M, Higuchi N, Oda M, Endo S, et al. Clinical and endoscopic characteristics of acute haemorrhagic rectal ulcer, and endoscopic haemostatic treatment: a retrospective study of 95 patients. Colorectal Dis. 2010;12(10 Online):e320-325. 